\title{
BMJ Open A randomised comparison of Conventional versus Intentional straTegy in patients with high Risk prEdiction of Side branch OccLusion in coronary bifurcation interVEntion: rationale and design of the CIT- RESOLVE trial
}

\author{
Dong Zhang, ${ }^{1,2}$ Dong Yin, ${ }^{1,2}$ Chenxi Song, ${ }^{1,2}$ Chengang Zhu, ${ }^{1,2}$ Ajay J Kirtane, ${ }^{3}$ \\ Bo Xu, ${ }^{1,2}$ Kefei Dou ${ }^{1,2}$
}

To cite: Zhang D, Yin D, Song C, et al. A randomised comparison of Conventional versus Intentional straTegy in patients with high Risk prEdiction of Side branch OccLusion in coronary bifurcation interVEntion: rationale and design of the CIT-RESOLVE trial. BMJ Open 2017;7:e016044. doi:10.1136/ bmjopen-2017-016044

- Prepublication history and additional material are available. To view these files please visit the journal online (http://dx.doi. org/10.1136/bmjopen-2017016044).

DZ and DY contributed equally.

Received 25 January 2017 Revised 10 May 2017 Accepted 10 May 2017

CrossMark

For numbered affiliations see end of article.

Correspondence to

Prof Bo Xu; bxu@citmd.com and

Dr Kefei Dou;

drdoukefei@126.com

\section{ABSTRACT}

Introduction The intentional strategy (aggressive side branch (SB) protection strategy: elective two-stent strategy or jailed balloon technique) is thought to be associated with lower SB occlusion rate than conventional strategy (provisional two-stent strategy or jailed wire technique). However, most previous studies showed comparable outcomes between the two strategies, probably due to no risk classification of SB occlusion when enrolling patients. There is still no randomised trial compared the intentional and conventional strategy when treating bifurcation lesions with high risk of SB occlusion. We aim to investigate if intentional strategy is associated with significant reduction of SB occlusion rate compared with conventional strategy in high-risk patients.

Methods and analysis The Conventional versus Intentional straTegy in patients with high Risk prEdiction of Side branch OccLusion in coronary bifurcation interVEntion (CIT-RESOLVE) is a prospective, randomised, singleblind, multicentre clinical trial comparing the rate of SB occlusion between the intentional strategy group and the conventional strategy group (positive control group) in a consecutive cohort of patients with high risk of side branch occlusion defined by V-RESOLVE score, which is a validated angiographic scoring system to evaluate the risk of SB occlusion in bifurcation intervention and used as one of the inclusion criteria to select patients with high SB occlusion risk (V-RESOLVE score $\geq 12$ ). A total of 21 hospitals from 10 provinces in China participated in the present study. 566 patients meeting all inclusion/exclusion criteria are randomised to either intentional strategy group or conventional strategy group. The primary endpoint is SB occlusion (defined as any decrease in thrombolysis in myocardial infarction flow grade or absence of flow in SB after main vessel stenting). All patients are followed up for 12-month postdischarge.

Ethics and dissemination The protocol has been approved by all local ethics committee. The ethics committee have approved the study protocol, evaluated

\section{Strengths and limitations of this study}

- Conventional versus Intentional straTegy in patients with high Risk prEdiction of Side branch OccLusion in coronary bifurcation interVEntion (CIT-RESOLVE) is the leading trial that intends to investigate if intentional strategy could decrease the rate of side branch (SB) occlusion in patients with high risk of SB occlusion.

- This study enrols high-risk patients by using an inclusion criteria of SB occlusion risk (V-RESOLVE score $\geq 12$ points).

> This study would provide evidence for interventionalists in strategy selection when treating bifurcation with high risk of SB occlusion.

- Not all bifurcation lesions are included in the present study; left main diseases are excluded.

the risk to benefit ratio, allowed operators with a minimum annual volume of 200 cases to participate in the percutaneous coronary intervention procedure and permitted them to perform both conventional and intentional strategies. Written informed consent would be acquired from all participants. The findings of the trial will be shared by the participant hospitals and disseminated through peer-reviewed journals.

Trial registration number NCT02644434; Pre-results.

\section{INTRODUCTION}

Approximately $15 \%$ to $20 \%$ of percutaneous coronary interventions (PCI) are performed to treat coronary bifurcation lesions. ${ }^{1-3}$ Previous studies have shown similar short-term and long-term clinical outcomes between the conventional strategy (eg, provisional two-stent strategy or jailed wire technique ${ }^{4-6}$ ) and the 
intentional strategy (eg, elective two-stent strategy or jailed balloon technique) $7^{78}$ thus, the conventional strategy is generally preferred for its easy use and reduced procedure time. However, the optimal interventional strategy selection for complex coronary bifurcation lesions remains somewhat controversial because of the variability in side branch (SB) disease and the desire to preserve patency of large diseased SBs. SB occlusion after main vessel (MV) stenting is one of the most serious complications during the procedure and may be the major reason why operators prefer more aggressive strategy in the complex bifurcation lesions. Our study has shown that the rate of SB occlusion was $7.37 \%$ in patients underwent conventional strategy, ${ }^{9}$ which was in accordance with previous studies (SB occlusion rate: $8.4 \%-19 \%){ }^{10-12} \mathrm{SB}$ occlusion can result in vessel closure and ischaemia, with clinically significant myocardial infarction (MI) and even death depending on the size of the SB (and the myocardial territory subtended by it). ${ }^{12} 13$

The risk and incidence of SB occlusion are important factors impacting the interventional strategy selection and clinical outcome. ${ }^{9}$ However, since the lack of useful tool for risk prediction of SB occlusion, no previous studies have considered the risk of SB occlusion as one of the inclusion criteria during patient enrolment. Previous randomised clinical trials performed randomisation of all categories of bifurcation lesions by using computer-generated random sequence totally ignored the individual lesion anatomical characteristics and the risk of SB occlusion. Now, we have developed an angiographic tool for risk prediction of SB occlusion, the Visual estimation for Risk prEdiction of Side branch OccLusion in coronary bifurcation interVEntion (V-RESOLVE) score, which can help risk stratification of SB occlusion and could also be used as a tool to select highrisk patients in randomised study. The SB occlusion rate was significantly higher in the high-risk group (V-RESOLVE score $\geq 12$, rate of SB occlusion: $16.7 \%$ ) than the non-highrisk group (V-RESOLVE score $<12$, rate of SB occlusion: $4.3 \%)$ as assessed by the V-RESOVLE score. ${ }^{14}$

Bifurcation lesions with high risk of SB occlusion may need intentional interventional strategy, which is more aggressive in SB protection than conventional strategy and considered to be associated with lower SB occlusion rate. However, no randomised trials were performed to compare the rate of SB occlusion between intentional strategy and conventional strategy in high-risk patients.

Accordingly, the present study is designed to enrol patients with high risk of SB occlusion (V-RESOLVE score $\geq 12$ ) and investigate if intentional strategy is associated with significant reduction of SB occlusion rate compared with conventional strategy in patients with high risk of SB occlusion.

\section{METHODS AND ANALYSIS}

\section{Hypothesis to be tested}

We hypothesised that for patients at high risk of SB occlusion (V-RESOLVE score $\geq 12$ ), intentional strategy (a more aggressive SB protection strategy: elective two-stent strategy or jailed balloon technique) is associated with significant reduction of SB occlusion rate compared with conventional strategy (provisional two-stent strategy or jailed wire technique). Thus, the hypothesis to be decided on are as follows: $\mathrm{H}_{0}$, for patients with high risk prediction of SB occlusion, there is no difference in the rate of SB occlusion between intentional strategy group and the conventional strategy group, versus $\mathrm{H}_{1}$, the rate of $\mathrm{SB}$ occlusion in intentional strategy group would be significantly lower than that of conventional strategy group.

\section{Study design}

The CIT-RESOLVE is a prospective, randomised (1:1), single-blind, multicentre clinical trial comparing the rate of SB occlusion between the conventional strategy group and the intentional strategy group in a consecutive cohort of high-risk coronary bifurcation patients. Although operators are not blinded, all individuals analysing the data are masked to treatment assignment. A total of 21 centres in China will enrol patients. This study is registered on www.clinicaltrials.gov, and the registration number is NCT 02644434 . The study flowchart is shown in figure 1 and its legend.

This trial is conducted in accordance with the Declaration of Helsinki and good clinical practice guidelines. The conduct of the trial has been approved by the ethics committee. Written informed consent would be acquired from all participants. Patient data in the Data Management System are protected by password and only available to users designated by the study with appropriate authorisation levels. Deidentified data will be used for data analysis.

\section{Risk prediction of SB occlusion}

V-RESOLVE score would be used for risk prediction of SB occlusion. The RESOLVE (Risk prEdiction of Side branch OccLusion in coronary bifurcation interVEntion) score, which is developed on the basis of quantitative coronary angiography (QCA), is a validated angiographic scoring system to evaluate the risk of $\mathrm{SB}$ occlusion in bifurcation intervention. ${ }^{9}$ The QCA-based RESOLVE score system contains six independent risk factors of SB occlusion: including two visual estimation predictors (plaque distribution and MV thrombolysis in MI (TIMI) flow grade before stenting) and four QCA analysis predictors (preprocedural diameter stenosis of bifurcation core, bifurcation angle, diameter ratio between $\mathrm{MV} / \mathrm{SB}$ and diameter stenosis of SB before MV stenting).

Although QCA provides a more objective determination of the extent and severity of coronary artery disease, it may be more time-consuming and/or not immediately available in real-time. As a result, the inclusion of QCA data within the QCA-based RESOLVE score limits its ability to be used at the time of bifurcation intervention. ${ }^{15}$ Therefore, we evaluated the ability of a visually estimated RESOLVE (V-RESOLVE) score to predict the risk of SB occlusion during bifurcation intervention. We found that the V-RESOLVE score, an easy-to-use score system based on visual estimation, can help risk stratification of SB occlusion 


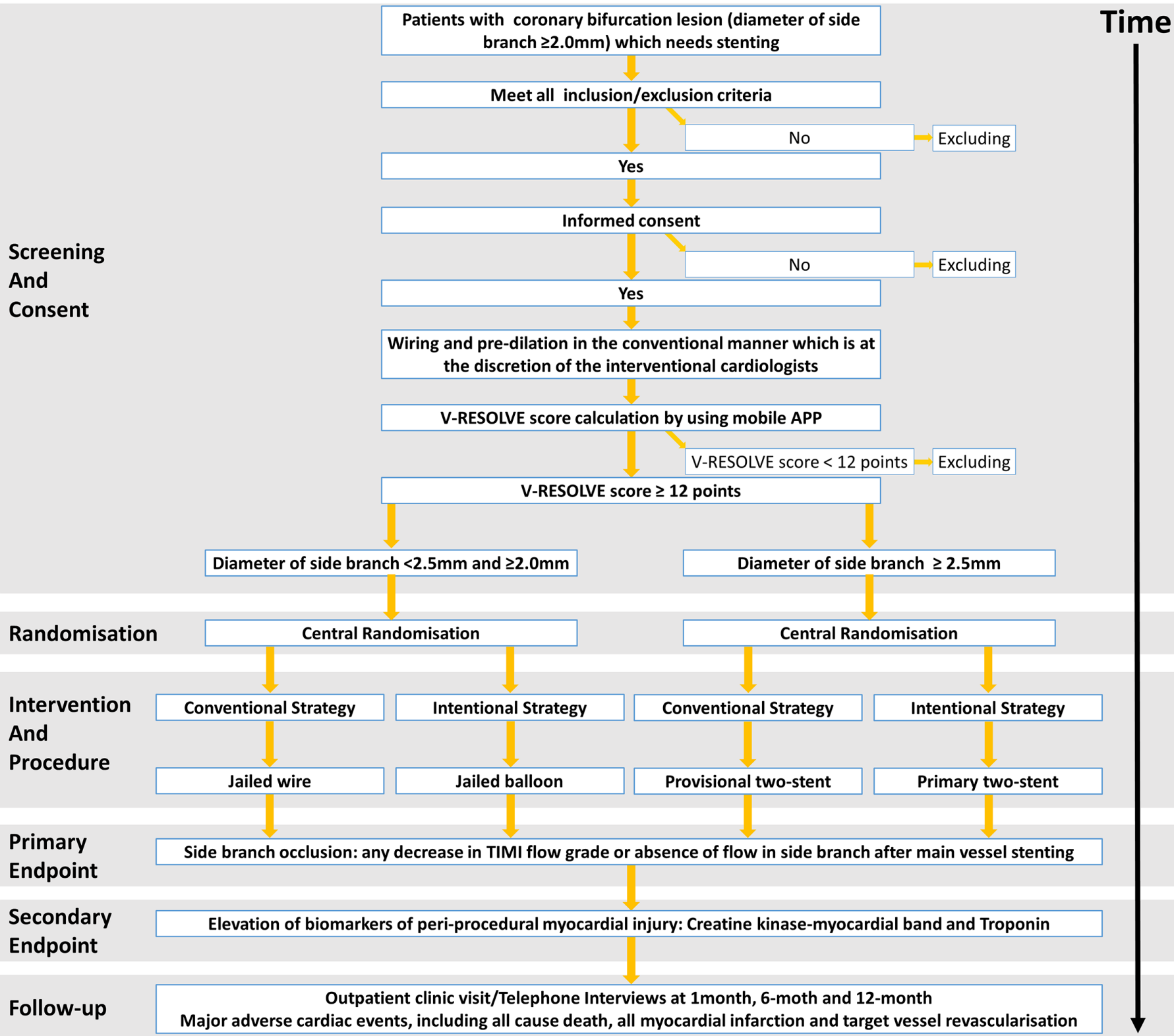

Figure 1 Study flowchart screening, randomisation, intervention, procedure, study endpoint and follow-up of CIT-RESOLVE trial. CIT-RESOLVE, Conventional versus Intentional straTegy in patients with high Risk prEdiction of Side branch OccLusion in coronary bifurcation interVEntion.

during coronary bifurcation intervention. The rate of SB occlusion was significantly higher in high-risk group (V-RESOLVE score $\geq 12$, rate of $\mathrm{SB}$ occlusion: $16.7 \%$ ) than that in non-high-risk group (V-RESOLVE score $\leq 11$, rate of SB occlusion: $4.3 \%)(\mathrm{p}<0.01)$. V-RESOLVE score makes precision medicine possible in the daily practice of coronary bifurcation intervention for its easy use. The development, validation and calculation methods are detailed in our previous study. ${ }^{14}$ The V-RESOLVE score is calculated by using a dedicate app, which is available in both the iTunes store and Google Play Store. Only patients with V-RESOLVE score $\geq 12$ would be enrolled.

\section{Study population}

A total of 566 patients with coronary bifurcation lesions (at high risk of SB occlusion), requiring PCI with stent implantation, are studied. V-RESOLVE score $\geq 12$ points are defined as lesions with high risk of SB occlusion. This implies the application of only few angiographic exclusion criteria (table 1). All patients provide written informed consent.

\section{Hospitals selection}

A total of 21 hospitals from 10 provinces (Beijing, Shanghai, Guangdong, Shanxi, Heilongjiang, Jilin, Liaoning, Guangxi, Hunan and Hebei, detailed in online supplementary file) are chosen. The annual PCI volume of each of these hospitals is $\geq 800$. Only operators with a minimum annual volume of 200 cases are allowed to participate in the PCI procedure. All these interventionalists are skilled in coronary bifurcation PCI and qualified to perform both conventional and intentional strategies. 
Table 1 Inclusion and exclusion criteria

\section{Inclusion criteria}

Clinical inclusion criteria:

- Subject must be male or non-pregnant female $\geq 18$ years of age and $\leq 75$ years of age;

- Subject has symptomatic coronary artery disease with objective evidence of ischaemia or silent ischaemia;

- Subject is eligible for $\mathrm{PCl}$;

- Subject (or legal guardian) understands the trial requirements and the treatment procedures and provides written informed consent before any trial-specific tests or procedures are performed;

- Subject is willing to comply with all protocol-required followup evaluation.

\section{Angiographic inclusion criteria:}

- Subjects have coronary bifurcation lesions requiring PCI with stent implantation according to clinical guidelines and/ or the operator's judgement;

- Visually estimated reference vessel diameter (RVD) of target main vessel $\geq 2.5 \mathrm{~mm}$ and $\leq 4.0 \mathrm{~mm}$;

- Visually estimated RVD of target side branch $\geq 2.0 \mathrm{~mm}$;

- Coronary anatomy is likely to allow delivery of a study device to the target lesion(s);

- V-RESOLVE score $\geq 12$ points.

\section{Exclusion criteria}

Clinical exclusion criteria:

- Subject has a known allergy to contrast (that cannot be adequately premedicated) and/or the trial stent system or protocol-required concomitant medications (eg, stent alloy, stainless steel, sirolimus, everolimus or structurally related compounds, polymer or individual components, all P2Y inhibitors or aspirin);

- Planned surgery within 6 months after the index procedure;

- Subject has one of the following (as assessed prior to the index procedure):

- Other serious medical illness (eg, cancer, congestive heart failure) with estimated life expectancy of less than 12 months;

- Current problems with substance abuse (eg, alcohol, cocaine and heroin);

- Planned procedure that may cause non-compliance with the protocol or confound data interpretation.

- Subject has a history of bleeding diathesis or coagulopathy or will refuse blood transfusions;

- Subject is participating in another investigational drug or device clinical trial that has not reached its primary endpoint;

- Subject intends to participate in another investigational drug or device clinical trial within 12 months after the index procedure;

- Subject with known intention to procreate within 12 months after the index procedure (women of childbearing potential who are sexually active must agree to use a reliable method of contraception from the time of screening through 12 months after the index procedure);

- Subject is a woman who is pregnant or nursing (a pregnancy test must be performed within 7 days prior to the index procedure in women of childbearing potential);

- Subject with left ventricular ejection fraction $<35 \%$;

- Subject has preoperative renal dysfunction: serum creatinine $>2.0 \mathrm{mg} / \mathrm{dL}(176.82 \mu \mathrm{mol} / \mathrm{L})$.

Angiographic exclusion criteria:

Left main lesions;

- In case of acute myocardial infarction (MI) of which the culprit vessel located at the left anterior descending coronary artery (LAD), the bifurcation lesion (LAD/diagonal branch (RVD $>2.5 \mathrm{~mm}$ )), which is proximal to occluded LAD segment, should be excluded.

$\mathrm{PCl}$, percutaneous coronary intervention.

\section{Investigator training}

All investigators received comprehensive training on the standard definition of elements, protocol, app using, calculation of V-RESOLVE score, randomisation, standard procedure of PCI and data management.
Although there are only six variables in the V-RESOLVE score, intraobserver and interobserver variability for visual estimation is always a question for every visual score system and is also a major concern of us. To minimise the intraobserver and interobserver variability in the calculation of V-RESOLVE score, all investigators 
have undergone an extensive training session by a group of experienced technicians from the angiographic core laboratory in Fuwai Hospital on 13 August 2016. The training session included: (1) calculate the V-RESOLVE score of low-risk and high-risk bifurcation lesions and (2) a comprehensive review of bias, discrepancies and pitfalls related to these cases. The investigator interobserver agreement was found to be substantial or greater (Fleiss Kappa $>0.60)$ after training. Once the investigators are not sure that the V-RESOLVE score is $\geq 12$ points or not, we recommend them to send the cineangiograms by internet to the angiographic core laboratory in Fuwai Hospital, where cineangiograms would be assessed by two experienced technicians together, and the V-RESOLVE score was generated by consensus.

\section{Patient enrolment and randomisation}

Subjects must be $\geq 18$ years and $\leq 75$ years of age at the time of enrolment in the study. Coronary angiography would be performed to confirm that angiographic inclusion criteria are met. Then, wiring and predilation would be performed at the discretion of the interventional cardiologists in the conventional manner. A mobile app specialised for V-RESOLVE calculation will be used to calculate the V-RESOLVE score after predilation. Bifurcation lesions with V-RESOLVE score of $\geq 12$ points will be enrolled. Patients that meet all the inclusion criteria and have no exclusion criteria would be included in this study. Patient enrolment has been started on 1 December 2016 and anticipated to be completed before December 2017.

Patient randomisation will be performed centrally by internet after signing an informed consent form. The randomisation will be stratified by the diameter of $\mathrm{SB}$ (diameter of $\mathrm{SB}<2.5 \mathrm{~mm}$ and $\geq 2.0 \mathrm{~mm}$ vs diameter of $\mathrm{SB} \geq 2.5 \mathrm{~mm}$ ), with a randomisation ratio of $1: 1$ to either conventional strategy group or intentional strategy group.

\section{Intervention and procedure}

PCI is undertaken via the access site of operators' choice. Coronary angioplasty is performed in the conventional manner and coronary stents or other procedures/ devices are used only when required. The administration of periprocedural antiplatelet and antithrombotic medications is based on the operator's discretion and current guidelines. Intravenous unfractionated heparin is used to maintain an activated clotting time between $250 \mathrm{~s}$ and $300 \mathrm{~s}$ through the whole procedure. Cardiac enzymes (creatine kinase-myocardial band (CK-MB) and troponin) are dynamically measured until 48 hours postprocedure. Lifelong aspirin $(100 \mathrm{mg} / \mathrm{d})$ is prescribed to all patients. At least 12 months of clopidogrel $(75 \mathrm{mg} / \mathrm{d})$ would be recommended to all patients.

\section{Conventional strategy group}

Patients randomised to the conventional strategy group would undergo either jailed wire technique (diameter of $\mathrm{SB}<2.5 \mathrm{~mm}$ and $\geq 2.0 \mathrm{~mm}$ ) or provisional two-stent strategy (diameter of $\mathrm{SB} \geq 2.5 \mathrm{~mm}$ ).
Jailed wire technique

Both MV and SB are wired, with lesion preparation at the operator's discretion. The MV is stented with wire protection in SB. The SB is not further treated unless there is threatened $\mathrm{SB}$ closure, severe ostial pinching of SB $(>90 \%)$, TIMI flow grade decrease in SB, or SB dissection greater than type A. If one of these criteria exists, the SB would be rewired and a kissing balloon inflation is undertaken with anatomically appropriate sizing for each vessel.

\section{Provisional two-stent strategy}

Both vessels are wired, with lesion preparation and MV stenting the same as the jailed wire technique. Provisional $\mathrm{T}$ stenting of the SB could be undertaken if one of the following criteria exists after $\mathrm{SB}$ rewiring and a kissing balloon inflation is undertaken: threatened SB closure, severe ostial pinching of SB $(>90 \%)$, TIMI flow grade decrease in $\mathrm{SB}$, or $\mathrm{SB}$ dissection greater than type A.

\section{Intentional strategy group}

In the present trial, we would enrol high-risk SB with diameter $\geq 2.0 \mathrm{~mm}$, which would critically impact the prognosis. However, elective two-stent strategy is not appropriate for all SB with diameter $\geq 2.0 \mathrm{~mm}$. Thus, we use two aggressive strategies in intentional strategy group: jailed balloon technique (for $\mathrm{SB}$ with diameter $<2.5 \mathrm{~mm}$ and $\geq 2.0 \mathrm{~mm}$ ) or elective two-stent strategy (for SB with diameter $\geq 2.5 \mathrm{~mm}$ ).

\section{Jailed balloon technique}

The technique has been detailed in previous studies. ${ }^{45}$ To be brief, vessel wiring and lesion preparation are the same as the jailed wire technique. A balloon that is appropriately sized to approximate the reference vessel diameter (RVD) of $\mathrm{SB}$ is advanced into the SB. A stent is then advanced into correct position over the target lesion in the MV. To prevent entrapment of the SB balloon, the proximal marker of the balloon is positioned approximately $2 \mathrm{~mm}$ proximal to the MV stent. Adequate length of balloon is advanced into SB to project the ostium. Then, the stent in MV is deployed to nominal pressures, jailing the SB balloon and wire. If the $\mathrm{SB}$ is not compromised, then the jailed SB balloon is inflated to low pressure $(<3$ atmospheres), deflated, and the SB wire and balloon removed. Then the SB is rewired, followed by mandatory proximal optimisation technique.

However, if there is TIMI flow grade decrease in SB, the balloon is inflated to try to reopen the SB. After the $\mathrm{SB}$ is rewired, the $\mathrm{SB}$ balloon is removed. Ballooning or $\mathrm{T}$ stenting of the SB could be undertaken. POT is mandated to achieve good apposition of the proximal MV stent after the $\mathrm{SB}$ is reopened. The wire in SB will not be removed until the POT is completed.

No matter there is SB compromise or not, final kissing balloon technique could be performed at the discretion of the interventional cardiologists. 


\section{Elective two-stent strategy}

Patients in this subgroup would undergo crush procedure (eg, Crush, Balloon Crush or DK-Crush) ${ }^{16-18}$ or any other elective two-stent strategy like Culotte and T stent, ${ }^{19} 20$ which stenting SB before MV stenting. These techniques were detailed in previous studies. ${ }^{16-20}$

For both the conventional and intentional strategy groups, proximal or distal dissections could be treated with further stenting at any stage. Post-dilations could be performed to optimise stent expansion. In all cases, an additional vessel with other lesions could be treated if required.

\section{Primary and secondary endpoint(s)}

The primary endpoint is SB occlusion, which is defined as any decrease in TIMI flow grade or absence of flow in SB after MV stent well opposed. For lesions underwent conventional strategy, TIMI flow grade is assessed immediately after the MV stent is deployed and post-dilation (if post-dilation is performed), then, the SB could be further treated if required. For lesions underwent jailed balloon technique, TIMI flow grade is assessed after POT is performed. For lesions underwent elective two-stent strategy, TIMI flow grade is assessed immediately after the MV stent is deployed and post-dilation (if post-dilation is performed), then rewiring the SB or final kissing balloon is performed if required.

The secondary endpoints are: (1) the elevation of biomarkers of periprocedural myocardial injury (CK-MB and troponin); periprocedural MI is defined as biomarkers elevation $\geq 10 \times$ upper reference limit (URL) for CK-MB and/or $\geq 70 \times$ URL for troponin ${ }^{21}$ and (2) 12-month major adverse cardiac events (MACE, including all-cause death, all MIs and target vessel revascularisation).

\section{Follow-up}

Subjects had either a telephone call or clinic visit at 30 days ( \pm 7 days), 3 months ( \pm 14 days), 6 months ( \pm 14 days) and 12 months ( \pm 30 days) by the enrolling site for outcome evaluation. For all patients, MACE at 12 months will be reported. MACE will be defined as a composite of all-cause death, all MIs (defined by the Third Universal Definition ${ }^{22}$ ) and target vessel revascularisation (defined by the Academic Research Consortium $^{23}$ ).

\section{Data collection}

Profession trained staff who are independent of patient treatment will be responsible for data collection and entering. The data collected for each new CIT-RESOLVE patient include baseline information, sociodemographic characteristics, symptoms and signs of the presenting coronary disease, medical history, biomarker findings (CK-MB and troponin activity will be determined by using an immunoinhibition assay and confirmed by mass spectrometry), electrocardiography, and treatments administered prior to admission during hospitalisation. Final diagnosis, major in-hospital clinical events (death, periprocedural MI, major bleeding and stroke) and discharge status will also be recorded.

Baseline and procedural coronary angiography will be reviewed and analysed by physicians and interventionalists to calculate the V-RESOLVE score. Coronary angiography findings, including bifurcation location, baseline and post-MV stenting TIMI flow grade in MV and SB, will be recorded. Procedural characteristics including interventional strategy, the presence of jailed wire/balloon and successful final kissing or not will be collected. All investigators are required to collect, recheck and input all these data and submit the completed electronic case report form on the patient's discharge or death. The investigation scheduling is detailed in table 2.

One follow-up survey (by outpatient clinic visit or telephone) will be conducted at 12 months after discharge, to collect information on medications, MACE and any rehospitalisations after discharge.

\section{Statistical considerations}

Sample size calculations

Sample size parameters for the primary endpoint:

- A 1:1 treatment allocation ratio of intentional strategy group and the conventional strategy group.

- A two-side significance level (alpha) of 0.05.

- Eighty per cent power to show differences in the rate of SB occlusion between intentional strategy group and conventional strategy group.

- The rate of $\mathrm{SB}$ occlusion in intentional strategy group: $4.0 \%$.

- The rate of SB occlusion in conventional strategy group: $10.0 \%$.

- The primary endpoint would be reached immediately after the MV stenting; therefore, the attrition rate is $0 \%$.

- Sample size formula:

$$
\mathrm{n}=\frac{\left[\mu_{1-\alpha / 2} \sqrt{2 \overline{\mathrm{p}}(1-\overline{\mathrm{p}})}+\mu_{1-\beta} \sqrt{\mathrm{p}_{\mathrm{T}}\left(1-\mathrm{p}_{\mathrm{T}}\right)+\mathrm{p}_{\mathrm{C}}\left(1-\mathrm{p}_{\mathrm{C}}\right)}\right]^{2}}{\left(\mathrm{p}_{\mathrm{T}}-\mathrm{p}_{\mathrm{C}}\right)^{2}}
$$

The $10 \%$ rate of SB occlusion in conventional strategy group is based on the V-RESOLVE study. ${ }^{15} \mathrm{It}$ is reasonable to assume that, with an intentional strategy for bifurcation lesions with V-RESOLVE score $\geq 12$ points, the rate of SB occlusion would decrease to $4 \%$ in intentional strategy group. Thus, the present study requires 283 subjects in intentional strategy group and 283 in conventional strategy group and the total number will be 566 .

\section{Analysis plan}

The statistical analyses of the full analysis set will follow the intention-to-treat (ITT) principle. The ITT set will consist of all subjects who signed the written informed consent and are randomised, regardless which strategy was selected. The primary analysis is a superiority ITT analysis of the primary clinical endpoint. Normal approximation test for the difference between two proportions (pooled proportion) or Fisher's exact test (if applicable) 

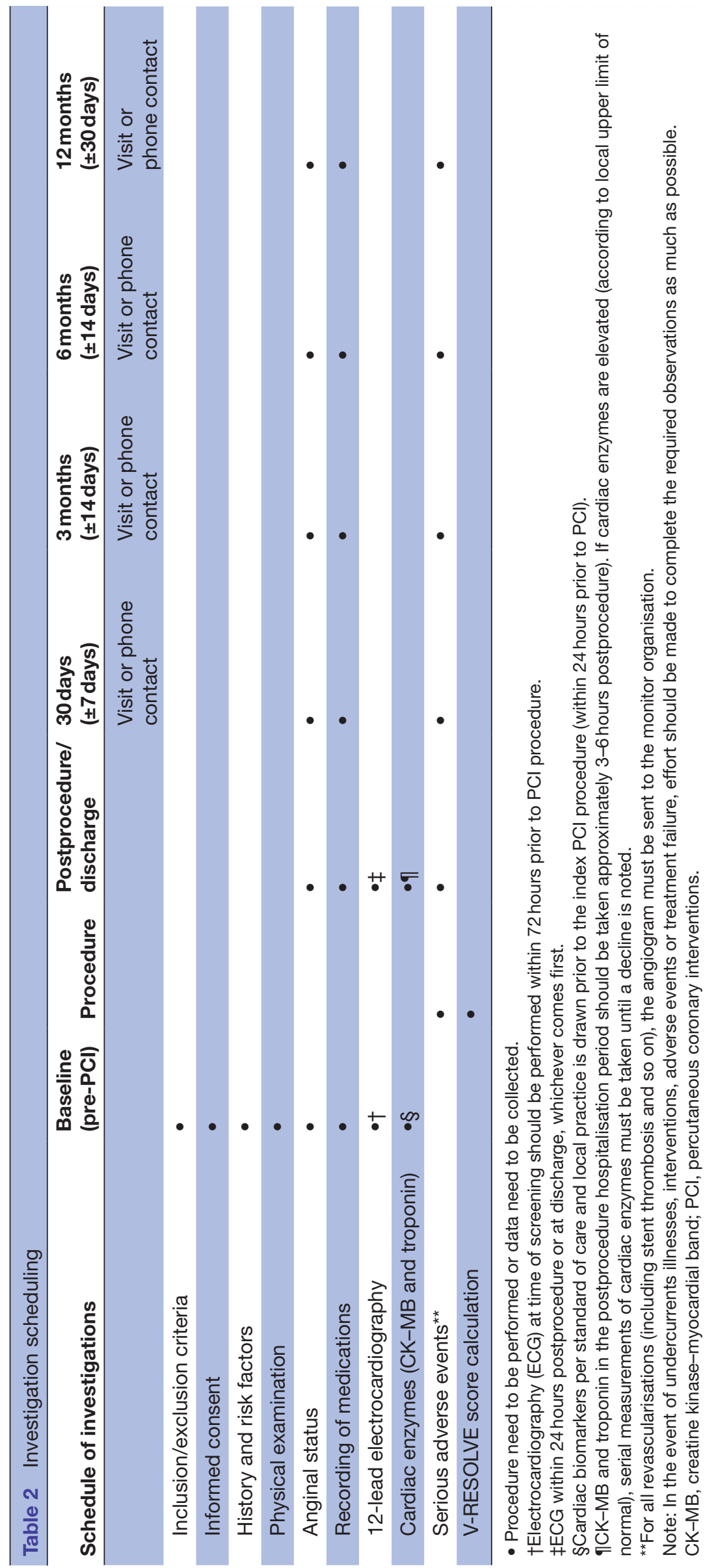
will be used to test the two-sided hypothesis of superiority in proportions. If the $\mathrm{p}$ value from the two-sided test is $<0.05$, the intentional strategy (test) will be concluded to be superior to conventional strategy. If required, an additional analysis of the per-protocol population will be conducted of the primary and secondary endpoints.

The conventional $\chi^{2}$ test or Fisher exact test will be used for the analysis of categorical variables. The treatment group differences will be evaluated with Student's t-test or Wilcoxon rank sum scores for continuous variables. The two strategies will be compared by Kaplan-Meier estimates for survival analysis. Statistical significance will be declared if the two-sided $p$ value is $<0.05$. All analyses will be performed with the use of the statistical programme SAS V.9.4.

\section{DISCUSSION}

During coronary bifurcation intervention, one of the most serious complications is SB occlusion. Keeping the $\mathrm{SB}$ open is the major principle during PCI. However, no previous randomised trials tried to address the problem of decreasing SB occlusion rate in patients with high risk of $\mathrm{SB}$ occlusion. The intentional strategy, which is more aggressive in SB protection, is thought to have lower SB occlusion rate. However, there is no concrete evidence confirming that intentional strategy is associated with significant reduction of SB occlusion rate compared with conventional strategy in patients with high risk of $\mathrm{SB}$ occlusion. CIT-RESOLVE is the leading randomised trial that attempts to clarify this issue. To the best of our knowledge, CIT-RESOLVE will be the first trial that (1) enrols high-risk patients by using an inclusion criteria of SB occlusion risk (V-RESOLVE score $\geq 12$ points) and (2) compares the rate of SB occlusion between intentional strategy and conventional strategy in patients with high risk of SB occlusion.

Series randomised clinical trials have attempted to address the problem of whether bifurcation lesions require stenting both the MV and $\mathrm{SB}$ or not. ${ }^{2} 6$ 19 24-33 However, the results of previous studies remain controversial: the BBC ONE study showed significant lower incidence of MACE in simple strategy group ${ }^{29}$ while the Double Kissing Crush (DKCRUSH) -II study showed a significant reduction of target lesion revascularisation and target vessel revascularisation in DK crush group. ${ }^{6}$ Most of the randomised clinical trials performed randomisation of all bifurcation lesions by using computer-generated random sequence, totally ignored the individual lesion anatomical characteristics and risk factors of SB occlusion. Thus, a substantial part of bifurcation lesions may not undergo proper intervention strategy though some patients have crossed over to another group. This may be the major reason why the results of previous studies remain controversial.

Previous studies enrolled patients by using the inclusion criteria of either unselected bifurcation lesions, specific Medina classifications or true bifurcation lesions.
However, neither 'Medina classification' nor 'true bifurcation lesion' could predict the risk of SB occlusion accurately. ${ }^{34}$ The SB occlusion risk is not considered as an important criterion when enrolling patients. CIT-RESOLVE is the first trial that only enrols high-risk patients by using a risk prediction tool (V-RESOLVE score $\geq 12$ points).

Numerous classifications and definitions of coronary bifurcation lesions have been proposed to simplify the hard topic of bifurcation lesion in interventional cardiology. ${ }^{36-45}$ Among them, 'Medina classification' as well as 'true bifurcation lesion' are straightforward and widely used. However, none of these classifications or definitions could accurately predict the risk of SB occlusion. ${ }^{35}$ One of our previous researches has shown that 'true bifurcation lesion' could not be regarded as an independent predictor of SB occlusion. ${ }^{34}$ RESOLVE score and V-RESOLVE score is the first attempt to stratify the risk of SB occlusion during coronary bifurcation intervention. V-RESOLVE score, which contains six independent predictors of SB occlusion, is a validated score system to evaluate the risk of SB occlusion ${ }^{14}$ and a useful tool for risk prediction of SB occlusion in the present study. V-RESOLVE score $\geq 12$ points is considered as high risk in SB occlusion, which may trigger interventional SB protection, and set as one of the inclusion criteria of CIT-RESOLVE trial.

The intentional strategy is more aggressive in SB protection: jailed wire may help SB reopen; stenting the SB before MV stenting may avoid SB occlusion. Thus, the intentional strategy is thought as a more suitable strategy for high-risk bifurcation lesion. CIT-RESOLVE is the leading trial that intends to investigate if intentional strategy could decrease the rate of $\mathrm{SB}$ occlusion in patients with high risk of SB occlusion. Comparing the rate of SB occlusion between intentional and conventional strategy would provide evidence for interventionalists in strategy selection when treating bifurcation with high risk of SB occlusion. Twelve-month follow-up would investigate if SB occlusion could impact the clinical outcome directly.

One limitation of the trial design is that not all highrisk bifurcation lesions are included in the present study. When treating left main diseases, left anterior descending artery or left circumflex artery occlusion may lead to serious outcome, thus, left main diseases are excluded in the consideration of ethic. Also, in case of acute MI of which the culprit vessel located at the LAD, the bifurcation lesion ( $\mathrm{LAD} /$ diagonal branch $(\mathrm{RVD}>2.5 \mathrm{~mm})$ ), which is proximal to occluded LAD segment, is excluded. Another limitation is that jailed balloon technique, which has not been proven by randomised clinical trials and widely used in clinical practice, is used in the interventional group. Although jailed balloon technique has been reported to be associated with very low rate of SB occlusion, ${ }^{4}$ its effect in SB protection warrant further studies. In future studies, we would compare the rate of SB occlusion between provisional two-stent strategy and elective two-stent strategy in patients at high risk of SB occlusion. 


\section{CONCLUSION}

The CIT-RESOLVE study is the first large randomised trial that enrols only high-risk patients by using an inclusion criteria of SB occlusion risk (V-RESOLVE score $\geq 12$ points), and it has sufficient power to assess the effect of intentional strategy in decreasing the $\mathrm{SB}$ occlusion rate in patients at high risk of SB occlusion.

\section{CIT-RESOLVE study group}

Principal investigator: KD (Fuwai Hospital and National Center for Cardiovascular Diseases). Co-principal investigator: BX (Fuwai Hospital and National Center for Cardiovascular Diseases). Coordinating center: Fuwai Hospital and National Center for Cardiovascular Diseases, Beijing, China. Advisory Chairmen: Yuejin Yang (Fuwai Hospital and National Center for Cardiovascular Diseases), Shaoliang Chen (Nanjing First Hospital and Nanjing Medical University) and AJK (Columbia University Medical Center and New York Presbyterian Hospital).

\section{Author affiliations}

${ }^{1}$ State Key Laboratory of Cardiovascular Disease, Cardiovascular Institute, Fuwai Hospital and National Center for Cardiovascular Diseases, Beijing, PR China ${ }^{2}$ Department of Cardiology, Cardiovascular Institute, Chinese Academy of Medical Sciences \& Peking Union Medical College, Beijing, PR China

${ }^{3}$ Columbia University Medical Center/New York Presbyterian Hospital, New York, New York, USA

Contributors All authors have contributed to the conception, critical review and revision process and have offered final approval of this manuscript.

Funding The CITCRESOLVE trial is physician initiated and supported by Investigator Sponsored Studies (Abbott Cardiovascular, Study No.: CORC10498) and Peking Union Medical College Youth Fund and Fundamental Research Funds for the Central Universities (grant number: 3332016130 ). The authors are solely responsible for the design and conduct of this study, all study analyses, the drafting and editing of the manuscript and its final contents.

Competing interests None declared.

Patient consent The present manuscript is protocol, no patient information is included.

Ethics approval The Ethics Committee of the Cardiovascular Institute and Fuwai Hospital.

Provenance and peer review Not commissioned; externally peer reviewed.

Data sharing statement The present manuscript is protocol, there is no additional unpublished data.

Open Access This is an Open Access article distributed in accordance with the Creative Commons Attribution Non Commercial (CC BY-NC 4.0) license, which permits others to distribute, remix, adapt, build upon this work non-commercially, and license their derivative works on different terms, provided the original work is properly cited and the use is non-commercial. See: http://creativecommons.org/ licenses/by-nc/4.0/

(C) Article author(s) (or their employer(s) unless otherwise stated in the text of the article) 2017. All rights reserved. No commercial use is permitted unless otherwise expressly granted.

\section{REFERENCES}

1. lakovou I, Schmidt T, Bonizzoni E, et al. Incidence, predictors, and outcome of thrombosis after successful implantation of drug-eluting stents. JAMA 2005;293:2126-30.

2. Steigen TK, Maeng M, Wiseth R, et al. Randomized study on simple versus complex stenting of coronary artery bifurcation lesions: the Nordic bifurcation study. Circulation 2006;114:1955-61.

3. Latib A, Colombo A. Bifurcation disease: what do we know, what should we do? JACC Cardiovasc Interv 2008;1:218-26.
4. Singh J, Patel Y, Depta JP, et al. A modified provisional stenting approach to coronary bifurcation lesions: clinical application of the "jailed-balloon technique". J Interv Cardiol 2012;25:289-96.

5. Burzotta F, Trani C, Sianos G. Jailed balloon protection: a new technique to avoid acute side-branch occlusion during provisional stenting of bifurcated lesions. Bench test report and first clinical experience. Eurolntervention 2010;5:809-13.

6. Chen SL, Santoso T, Zhang JJ, et al. A randomized clinical study comparing double kissing crush with provisional stenting for treatment of coronary bifurcation lesions: results from the DKCRUSH-II (Double Kissing Crush versus Provisional Stenting Technique for treatment of coronary bifurcation lesions) trial. J Am Coll Cardiol 2011;57:914-20.

7. Généreux P, Kumsars I, Lesiak M, et al. A randomized trial of a dedicated bifurcation stent versus provisional stenting in the treatment of coronary bifurcation lesions. J Am Coll Cardiol 2015;65:533-43.

8. Abdel-Latif A, Moliterno DJ. Bifurcation stenting techniques and outcomes in patients with stable coronary artery disease: more evidence suggesting simpler is safer. JACC Cardiovasc Interv 2015;8:561-3.

9. Dou K, Zhang D, Xu B, et al. An angiographic tool for risk prediction of side branch occlusion in coronary bifurcation intervention: the RESOLVE score system (Risk prEdiction of Side branch OccLusion in coronary bifurcation interVEntion). JACC Cardiovasc Interv 2015;8:39-46.

10. Kralev S, Poerner TC, Basorth D, et al. Side branch occlusion after coronary stent implantation in patients presenting with ST-elevation myocardial infarction: clinical impact and angiographic predictors. Am Heart J 2006;151:153-7.

11. Aliabadi D, Tilli FV, Bowers TR, et al. Incidence and angiographic predictors of side branch occlusion following high-pressure intracoronary stenting. Am J Cardiol 1997;80:994-7.

12. Hahn JY, Chun WJ, Kim JH, et al. Predictors and outcomes of side branch occlusion after main vessel stenting in coronary bifurcation lesions: results from the COBIS II Registry (COronary Blfurcation stenting). J Am Coll Cardiol 2013;62:1654-9.

13. Muramatsu T, Onuma Y, García-García HM, et al. Incidence and short-term clinical outcomes of small side branch occlusion after implantation of an everolimus-eluting bioresorbable vascular scaffold: an interim report of 435 patients in the ABSORB-EXTEND single-arm trial in comparison with an everolimus-eluting metallic stent in the SPIRIT first and II trials. JACC Cardiovasc Interv 2013;6:247-57.

14. Dou K, Zhang D, Xu B, et al. An angiographic tool based on visual estimation for risk prEdiction of Side branch OccLusion in coronary bifurcation interVEntion: the V-RESOLVE score system. Eurolntervention 2016;11:e1604-e1611.

15. Colombo A, Ruparelia N. When you ask yourself the question "should I protect the side branch?": the answer is "yes". JACC Cardiovasc Interv 2015;8:47-8.

16. Chen SL, Ye F, Zhang JJ, et al. [DK crush technique: modified treatment of bifurcation lesions in coronary artery]. Chinese Med J 2005;118:1746-50.

17. Lim PO, Dzavík V. Balloon crush: treatment of bifurcation lesions using the crush stenting technique as adapted for transradial approach of percutaneous coronary intervention. Catheter Cardiovasc Interv 2004;63:412-6.

18. Colombo A, Moses JW, Morice MC, et al. Randomized study to evaluate sirolimus-eluting stents implanted at coronary bifurcation lesions. Circulation 2004;109:1244-9.

19. Adriaenssens T, Byrne RA, Dibra A, et al. Culotte stenting technique in coronary bifurcation disease: angiographic follow-up using dedicated quantitative coronary angiographic analysis and 12-month clinical outcomes. Eur Heart J 2008;29:2868-76.

20. Sheiban I, Albiero R, Marsico F, et al. Immediate and long-term results of " $\mathrm{T}$ " stenting for bifurcation coronary lesions. Am J Cardiol 2000;85:1141-4.

21. Moussa ID, Klein LW, Shah B, et al. Consideration of a new definition of clinically relevant myocardial infarction after coronary revascularization: an expert consensus document from the Society for Cardiovascular Angiography and Interventions (SCAI). J Am Coll Cardiol 2013;62:1563-70.

22. Thygesen K, Alpert JS, Jaffe AS, et al. Third universal definition of myocardial infarction. J Am Coll Cardiol 2012;60:1581-98.

23. Cutlip DE, Windecker S, Mehran R, et al. Clinical end points in coronary stent trials: a case for standardized definitions. Circulation 2007;115:2344-51

24. Maeng M, Holm NR, Erglis A, et al. Long-term results after simple versus complex stenting of coronary artery bifurcation lesions: nordic Bifurcation Study 5-year follow-up results. J Am Coll Cardiol 2013;62:30-4. 
25. Sirker A, Sohal M, Oldroyd K, et al. The impact of coronary bifurcation stenting strategy on health-related functional status: a quality-of-life analysis from the BBC one (British Bifurcation Coronary; Old, New, and Evolving strategies) study. JACC Cardiovasc Interv 2013;6:139-45.

26. Song YB, Hahn JY, Song PS, et al. Randomized comparison of conservative versus aggressive strategy for provisional side branch intervention in coronary bifurcation lesions: results from the SMARTSTRATEGY (Smart Angioplasty Research Team-Optimal Strategy for Side Branch intervention in coronary bifurcation lesions) randomized trial. JACC Cardiovasc Interv 2012;5:1133-40.

27. Behan MW, Holm NR, Curzen NP, et al. Simple or complex stenting for bifurcation coronary lesions: a patient-level pooled-analysis of the nordic bifurcation study and the British bifurcation coronary study. Circ Cardiovasc Interv 2011;4:57-64.

28. Lin QF, Luo YK, Lin CG, et al. Choice of stenting strategy in true coronary artery bifurcation lesions. Coron Artery Dis 2010;21:345-51.

29. Hildick-Smith D, de Belder AJ, Cooter N, et al. Randomized trial of simple versus complex drug-eluting stenting for bifurcation lesions: the british bifurcation coronary study: old, new, and evolving strategies. Circulation 2010;121:1235-43.

30. Jensen JS, Galløe A, Lassen JF, et al. Safety in simple versus complex stenting of coronary artery bifurcation lesions. the nordic bifurcation study 14-month follow-up results. Eurolntervention 2008;4:229-33.

31. Colombo A, Bramucci E, Saccà S, et al. Randomized study of the crush technique versus provisional side-branch stenting in true coronary bifurcations: the CACTUS (Coronary bifurcations: application of the Crushing Technique using Sirolimus-Eluting stents) Study. Circulation 2009;119:71-8.

32. Ferenc M, Gick M, Kienzle RP, et al. Randomized trial on routine vs. provisional T-stenting in the treatment of de novo coronary bifurcation lesions. Eur Heart $J$ 2008;29:2859-67.

33. Pan M, de Lezo JS, Medina A, et al. Rapamycin-eluting stents for the treatment of bifurcated coronary lesions: a randomized comparison of a simple versus complex strategy. Am Heart J 2004;148:857-64.

34. Park TK, Park YH, Song YB, et al. Long-Term clinical outcomes of true and Non-True bifurcation lesions according to Medina classification- Results from the COBIS (COronary Blfurcation stent) II registry. Circ J 2015;79:1954-62.

35. Chen X, Zhang D, Yin D, et al. Can "true bifurcation lesion" actually be regarded as an independent risk factor of acute side branch occlusion after main vessel stenting?: A retrospective analysis of 1,200 consecutive bifurcation lesions in a single center. Catheter Cardiovasc Interv 2016;87(Suppl 1):554-63.

36. Medina A, Suárez de Lezo J, Pan M. [A new classification of coronary bifurcation lesions]. Rev Esp Cardiol 2006;59:183.

37. Y-Hassan S, Lindroos MC, Sylvén C. A novel descriptive, intelligible and ordered (DINO) classification of coronary bifurcation lesions. review of current classifications. Circ J 2011;75:299-305.

38. Movahed MR, Stinis CT. A new proposed simplified classification of coronary artery bifurcation lesions and bifurcation interventional techniques. J Invasive Cardiol 2006;18:99-204.

39. Lefèvre T, Louvard $Y$, Morice MC, et al. Stenting of bifurcation lesions: classification, treatments, and results. Catheter Cardiovasc Interv 2000;49:274-83.

40. Popma J, Bashore T. Qualitative and quantitative angiographyBifurcation lesions. Textbook of interventional cardiology. Philadelphia: WB Saunders, 1994:1055-8.

41. George BS, Myler RK, Stertzer SH, et al. Balloon angioplasty of coronary bifurcation lesions: the kissing balloon technique. Cathet Cardiovasc Diagn 1986;12:124-38.

42. Tsuchida $\mathrm{K}$, Colombo A, Lefèvre $\mathrm{T}$, et al. The clinical outcome of percutaneous treatment of bifurcation lesions in multivessel coronary artery disease with the sirolimus-eluting stent: insights from the arterial revascularization therapies Study part II (ARTS II). Eur Heart $J$ 2007;28:433-42.

43. RDS. Bifurcation lesions. the manual of interventional cardiology. 2001:233-43.

44. Dauerman HL, Higgins PJ, Sparano AM, et al. Mechanical debulking versus balloon angioplasty for the treatment of true bifurcation lesions. J Am Coll Cardiol 1998;32:1845-52.

45. Al Suwaidi J, Berger PB, Rihal CS, et al. Immediate and long-term outcome of intracoronary stent implantation for true bifurcation lesions. J Am Coll Cardiol 2000;35:929-36. 